\title{
JEM-X: The X-RAY MONITOR ON INTEGRAL
}

C. Budtz-Jørgensen ${ }^{a}$,N. Lund ${ }^{a}$, N. J. Westergaard ${ }^{a}$, S. Brandt ${ }^{a}$, A.Hornstrup ${ }^{a}$, I. Lundgaard Rasmussen $^{a}$, S. Laursen ${ }^{a}$, R. Kristensen ${ }^{a}$, P. B. Mogensen ${ }^{a}$, K. Harbo Andersen ${ }^{a}$, I. Rasmussen $^{a}$, J. Polny ${ }^{a}$, P. A. Jensen ${ }^{a}$, C. A. Oxborrow ${ }^{a}$, J. Chenevez $^{a}$ K. Om $\varnothing^{a}$, V.

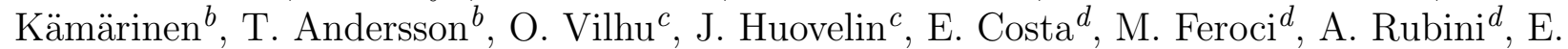
Morelli $^{d}$, A. Morbidini ${ }^{d}$, F. Frontera ${ }^{e}$, G. Zavattini ${ }^{e}$, V. Carassiti ${ }^{e}$, M. Morawski $^{f}$, G.

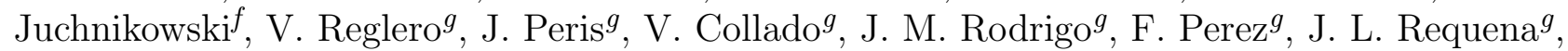
S. Larsson ${ }^{h}$, R. Svensson ${ }^{h}$, A. Zdziarski ${ }^{j}$, H. W. Schnopper ${ }^{k}$

${ }^{a}$ Danish Space Research Institute, Copenhagen, Denmark

${ }^{b}$ Metorex, Espoo, Finland

${ }^{c}$ University of Helsinki, Helsinki, Finland

${ }^{d}$ Instituto de Astrofisica CNR, Rome, Italy

${ }^{e}$ University of Ferrara, Ferrara, Italy

${ }^{f}$ Space Research Center, Warsaw, Poland

$g$ University of Valencia, Burjassot, Valencia, Spain

${ }^{h}$ Stockholm Observatory, Stockholm, Sweden

${ }^{i}$ Copernicus Astronomical Center, Warsaw, Poland

${ }^{k}$ Smithsonian Astrophysical Observatory, Cambridge, USA

\begin{abstract}
The INTEGRAL X-ray monitor, JEM-X, (together with the two gamma ray instruments, SPI and IBIS) provides simultaneous imaging with arcminute angular resolution in the 3-35 keV band. The good angular resolution and low energy response of JEM-X plays an important role in the detection and identification of gamma ray sources as well as in the analysis and scientific interpretation of the combined X-ray and gamma ray data. JEM-X is a coded aperture X-ray telescope consisting of two identical detectors. Each detector has a sensitive area of 500 $\mathrm{cm}^{2}$, and views the sky through its own coded aperature mask. The coded masks are located $3.4 \mathrm{~m}$ above the detector windows. The detector field of view is constrained by X-ray collimators $\left(6.6^{\circ}\right.$ FOV, FWHM).
\end{abstract}

Keywords: JEM-X, INTEGRAL, X-ray astronomy, Micro strip detector

\section{INTRODUCTION}

The primary instruments of the INTEGRAL payload are designed for detailed studies of celestial objects in the gamma ray region of the electromagnetic spectrum. The SPECTROMETER (SPI) covers the energy range 15 $\mathrm{keV}$ - $10 \mathrm{MeV}$ and the IMAGER (IBIS) $20 \mathrm{keV}-10 \mathrm{MeV}$.

To obtain a more complete picture of the physical conditions in the sources observed by the two main instruments, it is important to have simultaneous observations at both X-ray and optical wavelengths. The primary instruments are, therefore, augmented by an X-ray monitor, JEM-X, and an optical monitor, OMC. JEM-X provides spectral data in the $3-35 \mathrm{keV}$ band and also provides arcminute imaging to separate the contributions from close lying sources. The photon detection system consists of two identical imaging Microstrip Gas Chambers (MSGC) that view the sky through a coded aperture mask. Specifications describing JEM-X are given in Table 1. The present paper will summarize the most important features of the JEM-X telescopes and discuss the performance of the instruments both before and after they were launched into space. The technical details of the JEM-X detector have been described by Kämäräinen et al. (1997), ${ }^{1}$ and by Budtz-Jørgensen et al. (1997). ${ }^{2}$ Those for the JEM-X mask are given in a paper of Ballesteros et al. (1997),. ${ }^{3}$

Further author information: (Send correspondence to A.A.A.)

A.A.A.: E-mail: aaa@tbk2.edu, Telephone: 15051231234

B.B.A.: E-mail: bba@cmp.com, Telephone: +33 (0)1 987654 32, Address: A Very Long Address, City, Country 
Table 1. JEM-X specifications

\begin{tabular}{|c|c|}
\hline Mask diameter & $535 \mathrm{~mm}$ \\
\hline Detector diameter & $250 \mathrm{~mm}$ \\
\hline Mask-detector distance & $3401 \mathrm{~mm}$ \\
\hline Energy-range & $3-35 \mathrm{keV}$ Primary range \\
\hline Energy resolution & $\Delta \mathrm{E} / \mathrm{E}=0.40(\mathrm{E}[\mathrm{keV}])^{-1 / 2}$ \\
\hline Angular resolution & $3 \operatorname{arcmin}$ \\
\hline Field of view (diameter) & $\begin{array}{l}4.8^{\circ} \text { Fully coded } \\
7.5^{\circ} \text { Half response } \\
13.2^{\circ} \text { Zero response }\end{array}$ \\
\hline Point source location & $30 \operatorname{arcsec}($ for a $10 \sigma$ source) \\
\hline $\begin{array}{l}\text { Narrow line detection sensitivity } \\
\text { Isolated on-axis source } \\
\text { For a } 3 \sigma \text { detection in a } 10^{6} \text { s observation }\end{array}$ & $\begin{array}{l}1.710^{-5} \text { phot } \mathrm{cm}^{-2} \mathrm{~s}^{-2} @ 6 \mathrm{keV} \\
5.010^{-5} \text { phot } \mathrm{cm}^{-2} \mathrm{~s}^{-2} @ 30 \mathrm{keV}\end{array}$ \\
\hline $\begin{array}{l}\text { Continuum sensitivity } \\
\text { Isolated on-axis source } \\
\text { For a } 3 \sigma \text { detection in a } 10^{6} \mathrm{~s} \text { observation }\end{array}$ & $\begin{array}{l}1.410^{-5} \text { phot } \mathrm{cm}^{-2} \mathrm{~s}^{-2} @ 6 \mathrm{keV} \\
8.010^{-6} \text { phot } \mathrm{cm}^{-2} \mathrm{~s}^{-2} @ 30 \mathrm{keV}\end{array}$ \\
\hline Time resolution & $128 \mu \mathrm{s}$ \\
\hline
\end{tabular}

\section{CODED MASK}

The code patterns on the two coded masks are identical but the masks are turned $180^{\circ}$ with respect to each other to minimize common side lobes in the imaging process. The code is a hexagonal uniformly redundant array pattern based on the biquadratic residue set for the prime number 22501, in't Zand et. al.(1994) ${ }^{4}$ and Baumert (1971). ${ }^{5}$ This code is cut in a $0.5 \mathrm{~mm}$ thick tungsten plate. This thickness achieves opacities of $99.9 \%$ at 35 $\mathrm{KeV}$ and $95 \%$ at $60 \mathrm{KeV}$. The plates were manufactured using electro-discharge wire cutting which provides an accuracy of $0.01 \mathrm{~mm}$. The diameter of the coded area is $535 \mathrm{~mm}$ and the size of each hexagon is $3.3 \mathrm{~mm}$ (center to center distance). The mask/detector distance and mask element size defines the instrument angular resolution of 3.35 arcmin.

The basic mask pattern is larger than the detector window, therefore we do not have noise free coding. However, this configuration will minimize the sidelobes in the sky images.

Only $25 \%$ of the mask elements are open. A small value for the open fraction was chosen in order to achieve better source separation in crowded fields and also in an effort to save telemetry, which is very scarce on INTEGRAL.

The tungsten code plate is suspended under tension from a peripheral titanium ring. In order to withstand the accelerations during launch a titanium structure resembling a spider web is placed on both sides of the mask membrane. The loss of transparency due to this structure is less than $3 \%$ for on-axis sources. The masks were manufactured in Spain by SENER under the supervision of the University of Valencia. The total mass of each mask is $5.8 \mathrm{Kg}$.

\section{DETECTOR}

A photo of the JEM-X detector is shown in Fig. 2. It is a Micro strip Gas Chamber with a sensitive area of nominally $500 \mathrm{~cm}^{2}$. The detector consists of the following modules: the detector vessel, the collimator, the X-ray window and the Micro strip sensor package with the Detector Analog Electronics (DAE). The gas inside the detector is a mixture of xenon (90\%) and methane (10\%) at 1.5 bar pressure.

In total three full detector systems were fabricated, two of those, JEM-X1, JEM-X2 are now flying on INTEGRAL. The third detector is now used as a reference detector on-ground. 


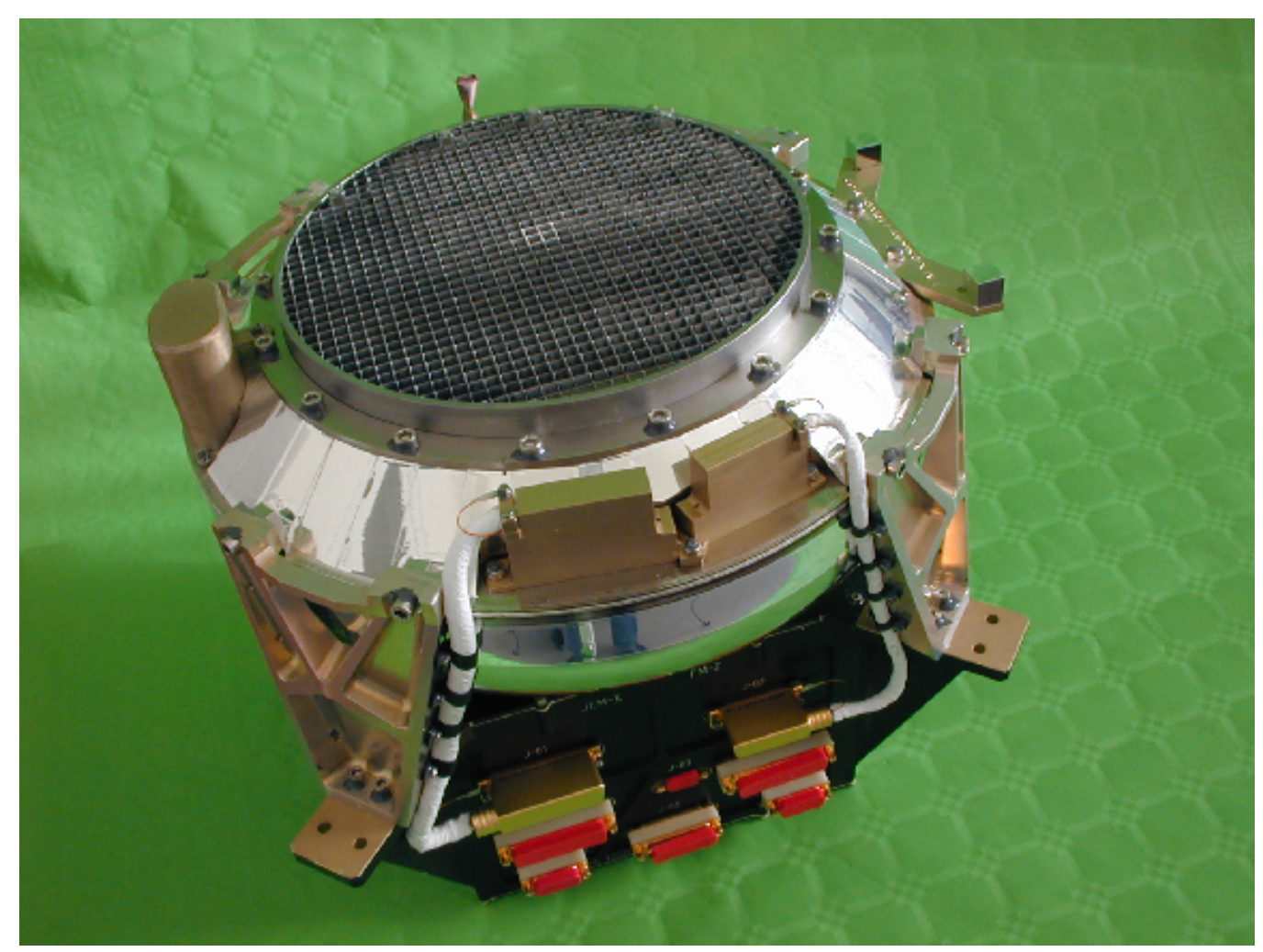

Figure 1. The JEM-X detector.

\subsection{Detector Structure}

The detector body is made of stainless steel and consists of two parts, the main- frame and the cover which are joined together by electron beam welding. There are no gaskets.

The cover is formed from a $2 \mathrm{~mm}$ thick stainless steel plate. The shape of the cover is similar to that of the cap for a pressure vessel.

The main-frame is a cone shaped ring with a circular flange in the middle for mounting the collimator. The gas filling tubes and vacuum tight signal connectors and high voltage feed troughs are welded to this main-frame. All internal structures are fixed to the main-frame. The inside structure consists of two sets of vertical studs and a spider structure carrying the 36 preamplifiers. The field forming rings are fixed by spacers on one set of studs and the microstrip-sensor package and the spider structure is mounted on the other set of studs.

\subsection{Collimator}

The collimator has a dual role, it acts as a support for the thin X-ray window against the internal pressure of the detector, and it limits the detector field of view. The full-width-at-half-maximum of the collimator field of view is tailored to have the same zero response as that of the detector-mask combination $\left(6.6^{\circ} \mathrm{FWHM}\right)$. This is the best match to maximize the instrument signal-to-noise ratio. The collimator cell geometry is chosen to be square. The core material of the collimator is molybdenum with a thickness of $180 \mu \mathrm{m}$. This allows to obtain a close to zero collimator response for incident angles greater than $6.6^{\circ}$. To reduce the fluorescence background the molybdenum it is covered by $35 \mu \mathrm{m}$ copper from both sides of the cell walls. Finally a $100 \mu \mathrm{m}$ aluminum layer will be added on top to absorb the $8 \mathrm{keV}$ K-fluorescence photons of copper.

Thus the collimator cells are made of crossed slats of molybdenum covered on both sides with a bilayer of copper and aluminium. The copper is fixed to the molybdenum plates with a double side cladding process. 
The aluminum layer is fixed on both sides by a diffusion bonding process. The single cells are stiffened with a eutectic $\mathrm{Zn}_{5} \mathrm{Al}$ brazing. The collimator was manufactured by Plansee, Austria under supervision of the University of Ferrara and Alenia.

The internal dimension of each cell is $6.6^{*} 6.6 \mathrm{~mm}$ and the height is $57.0 \mathrm{~mm}$. The cell assembly is circular with a diameter of $250 \mathrm{~mm}$ corresponding to the beryllium window diameter. The collimator mass is about $4.7 \mathrm{Kg}$. Four calibration sources are integrated within the collimator.

\subsection{Window}

The window is a $250 \mu \mathrm{m}$ thick beryllium foil. The window is supported by the collimator structure against the internal pressure. The window is glued to a stainless steel mounting ring which is welded to the detector main-frame. The window is electrically conductive and at the same electrical potential as the detector body.

\subsection{Microstrip Plate}

The Micro strip-sensor package consists of the following parts: The Micro strip plate, the support structure and preamplifiers and high voltage distribution circuits. All electronics inside the detector is mounted on ceramic circuit boards.

The capacitive read-out chains are mounted on ceramic plates which are glued on the Micro strip plate edges. The Micro strip electrodes are bonded to these chains. Signals from the capacitive chains are led through high voltage blocking capacitors to the preamplifiers on the ceramic circuit boards underneath the Micro strip plate.

The Micro strip pattern is shown schematically in Fig. 2. The pattern is chosen to be shaped as a regular octagon with a diameter of $292 \mathrm{~mm}$. The Micro strip pattern with alternating anode strips of $10 \mu \mathrm{m}$ and cathode strips of $458 \mu \mathrm{m}$ has a $1.062 \mathrm{~mm}$ pitch. The Micro strips are formed in a $0.2 \mu \mathrm{m}$ thick chromium layer sputtered on the substrate, D 263 glass(Schott). The cathode strips are connected to the printed circuit of the capacitive readout by wedge bonding to gold bonding pads on the chromium electrodes. The readout chains interconnect each cathode strip to the neighboring cathode strips by $2.2 \mathrm{nF}$ capacitors and $1 \mathrm{M} \Omega$ resistors.

The anode strips are connected into four groups, the signals from these anode segments are used for event triggering, energy determination and for pulse shape analysis. The cathode strips are read out to 11 amplifiers using the capacitive charge division chains. The position of the avalanche in the direction perpendicular to the micro-strip pattern is determined from the centroid of the avalanche charge. The orthogonal coordinate for an event is obtained from a set of pickup electrodes on the rear surface of the glass plate. These electrodes are arranged on a $2 \mathrm{~mm}$ pitch and read out by 20 amplifiers through capacitive chains.

The rear strips are surrounded by a veto-electrode which is used to suppress events caused by charged particles entering through the sides of the detector.

The detector requires two voltages in order to operate. The first is the drift voltage applied between the detector window (at ground potential) and the cathodes on the microstrip plate. The cathodes are biased positively with respect to the window, thus free electrons generated when an X-ray is absorbed in the xenon gas will drift to the microstrip plate by the drift field. The second voltage required by the detector is the gas-gain voltage. This voltage is applied between the anode and the cathode strips on the microstrip plate and controls the electron gas multiplication on the microtrip plate.

\subsection{Calibration sources}

The on board calibration system is composed of four collimated radioactive sources with a nominal strength of $25 \mathrm{Ci}$ (October 2002). The sources are placed within four cells of the JEM-X collimator. The ${ }^{109} \mathrm{Cd}$ sources are collimated using Au and Mo absorbers and are screwed into Al housings which are glued in their respective collimator cells. Each ${ }^{109} \mathrm{Cd}$ source emits 22,25 and $88 \mathrm{keV}$ photons and in addition they produce Ni fluorescence photons $(7.5,8.3 \mathrm{keV})$ from the source support Ni windows. The sources were provided by IAS, Rome. Two of the Cd sources for JEM-X1 were substituted by ${ }^{55} \mathrm{Fe}$ sources emitting $5.9 \mathrm{keV}$ photons.

The on-board calibration system is used to monitor the gas gain and thereby the energy calibration of the detectors. This is important especially during the first few hours after the High Voltage has been switched on where the microstrip gain changes (decreases) by $25 \%$. The gain is also affected by the detector temperature with a gain change of $1 \%$ per degree $\mathrm{C}$. 


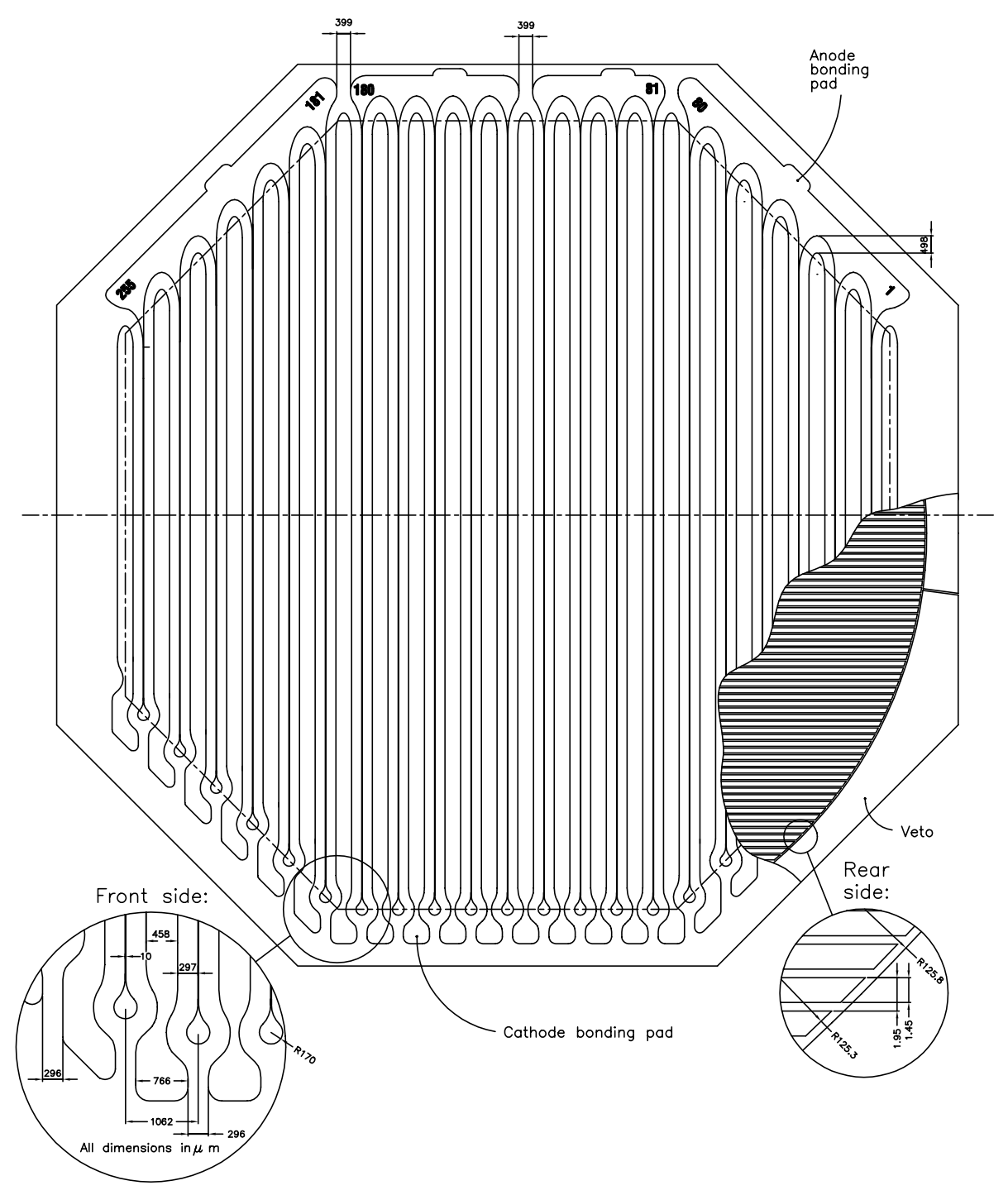

Figure 2. The JEM-X Microstrip plate.

\subsection{Electronics}

The instrument electronics is divided into two units, one unit (the "DFEE") which is specific for JEM-X, and another unit (the "DPE") which is very similar for all the instruments on INTEGRAL, and which is provided by ESA.

The DFEE incorporates a low voltage power supply, a dual high voltage unit (two voltages are needed for the JEM-X detector), 4 boards with the 34 amplifiers processing the signals from the detector, a housekeeping board, and a CPU board. All the electronics with the exception of the high voltage unit was build at DSRI. The $\mathrm{HV}$ unit was build by CAEN in Italy under supervision of IAS, Rome.

The timing of the analog event processing is controlled by an FPGA which also sends an interrupt to the processor for every new event. The FPGA also monitors the trigger rate from the detector and switches off the high voltage unit in case of very high counting rates. This mechanism has already been activated a number of times during the first months of the mission in connection with radiation belt entry. 


\section{ON-GROUND TEST AND CALIBRATION}

The JEM-X instruments underwent numerous test and calibration campaigns prior to the INTEGRAL launch. Only a summary and the most important results will be discussed here since the main focus will be on the in-flight experience.

\subsection{Detector life time tests}

It was a serious concern that the electronics mounted inside the active detector volume over time would contaminate the counter gas and thus degrade the detector energy resolution. Xenon based counter gasses are susceptible to contaminations down to $10 \mathrm{ppm}$ of water vapor or Oxygen, see i.e. Ramsey et al.(1988). ${ }^{6}$ Therefore all parts were carefully cleaned with solvents and baked before they were mounted in the detector vessel. The detectors were after the assembly evacuated and baked for 3 weeks at a temperature of $80^{\circ} \mathrm{C}$. The electronic components which were selected had previously been inserted in Xenon filled proportional wire chambers which were followed for at least a year to detect any sign of degradation due to gas contaminants. The assembled JEM-X detectors showed no signs of gas degradation over 1 year prior to launch, except for one detector for which a small gas leak was detected. The latter detector was repaired and serves now as an on-ground reference detector.

The fine anode strips of the microstrip plate are fragile and may be damaged if an electrical discharge is produced in the strong field around the anode strip. This effect limits gas gain for which the microstrip plate can be safely operated. The present microstrip design underwent a number of tests in order to establish safe operation voltages and gas gains. These investigations included measurements with an alpha particle source ${ }^{241} \mathrm{Am}$ inserted in the active detector volume in order to simulate the heavy ion flux of the cosmic ray particle background. The huge primary charge $\left(>10^{3}\right.$ times more than from an X-ray event) which a heavy cosmic ray particle crossing the detector might produce can generate an electrical breakdown in microstrip detectors, see Hott(1998) ${ }^{7}$ and Peskov et al.(1998). ${ }^{8}$ From our tests it was concluded that the detector filled with Xe $+10 \% \mathrm{CH} 4$ mixture at 1.5 bar can be safely operated at least up to a gas gain of 1500 . This gain is achieved at a voltage setting of $900 \mathrm{~V}$ between the cathode and anode strips. The gas gain of 1500 was chosen as the nominal gain value for the detectors and all on-ground calibrations were performed at this gain. Unfortunately, it was later discovered when the detectors were activated in space that the microstrip electrodes eroded severely at this gain and the gain had to be reduced by a factor 3 in order to assure sufficient life time ( 5 years) of the detectors, see discussion in the following section. The detectors were on ground and are in orbit operated with a drift voltage of $1100 \mathrm{~V}$, which creates a drift field of $200 \mathrm{~V} / \mathrm{cm}$ in the absorption volume between the detector entrance window and the microstrip plate.

\subsection{Instrument Calibrations}

The JEM-X detectors were calibrated at the X-ray facility at the University of Ferrara. This installation is described in Frontera et. $\mathrm{al}^{9}$ and consists of an X-ray generator, a monochromator and a XY table on which the detector is mounted such that the whole detector area could be scanned by the X-ray beam. The energy range of this equipment was from $15 \mathrm{keV}$ to $50 \mathrm{keV}$. An additional $5.9 \mathrm{keV}$ beam was provided by a collimated ${ }^{59} \mathrm{Fe}$ source. Including also the Xe K-escape peaks in the analysis it was possible to study the detector energy and position resolution down to $3 \mathrm{keV}$. The outcome of the calibration campaign is given in Westergaard et al. $(2002)^{10}$ and will only be summarized here.

A typical energy spectrum for the FM1 detector recorded with a beam energy of $40.15 \mathrm{keV}$ is shown in Fig. 3 . Besides the main line both the $\mathrm{Xe} \mathrm{K}_{\alpha}$ and $\mathrm{K}_{\beta}$ escape lines are clearly visible. The measured energy resolutions obey a $\sqrt{E}$ dependence with:

$$
\Delta E_{f w h m}=0.40 \sqrt{E[k e V]}
$$

where $\Delta \mathrm{E}_{f w h m}$ is the FWHM of the energy resolution. The detector gas gain varies smoothly over the microstrip plate with deviations of $\pm 10 \%$. These variations were mapped and corrections included on ground.

The measured detector position resolution (FWHM) was $2.0 \mathrm{~mm}$ at $3 \mathrm{keV}$ had a minimum value of $0.5 \mathrm{~mm}$ between 10 to $20 \mathrm{keV}$ and increased to $2 \mathrm{~mm}$ at $35 \mathrm{keV}$. The non linearities of detector position response was determined making fine scans of the full active detector area with X-ray beams of $0.5 \mathrm{~mm}$ diameter. The scans 


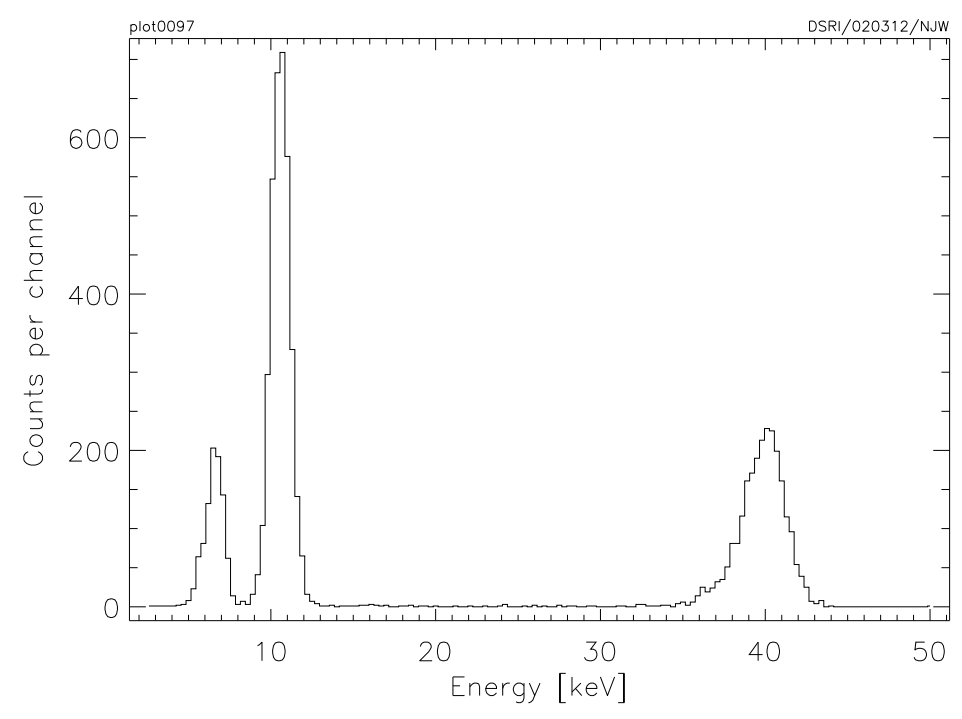

Figure 3. A spectrum recorded with the FM1 detector for a beam energy of $40.15 \mathrm{keV}$. The $\mathrm{K}_{\alpha}$ and $\mathrm{K}_{\beta}$ escape lines are clearly visible.

were performed at 3 energies $(5.9,17$ and $25 \mathrm{keV})$. Application of the derived position correction matrix reduces the systematic errors of the position determination to less than $0.2 \mathrm{~mm}$.

\section{JEM-X IN-FLIGHT PERFORMANCE}

The JEM-X detectors were activated about a week after the launch of INTEGRAL. The detectors were operated at the nominal gas gain of 1500. At first sight all detector systems operated and behaved as expected, however, after a few days it became apparent that the microstrip anodes eroded with an alarming rate of one anodestrip per day. As discussed in the previous section it is suspected that cosmic ray heavy ions can initiate electrical breakdowns close to the anodestrips. Apparently, the alpha particle tests conducted on ground did not realistically reflect the harsh conditions in space. This situation has forced changes in the operation of the detectors. The gas gain for both detectors has been reduced by a factor of 3 and at present the observations are executed with only one detector (JEM-X2) activated. The gain reduction diminished the damage rate to less than one anodestrip per month, a level for which the survival of the instruments should be assured for a five year period. The necessary gain reduction has affected the JEM-X performance to some degree, mainly because of the resulting decrease of the detector signal to noise ratios. This and further details on the in-orbit performance of JEM-X will be discussed in the following sections.

\subsection{Energy Range and Resolution}

The decrease in gas gain has modified the low energy threshold of the detectors. Fig. 4 compares the empty field energy spectrum obtained before the gain change (left) with the one measured after the gain change (right). There is a clear loss of events in the low energy part of the latter spectrum. A more detailed analysis has shown that the $50 \%$ efficiency level is now reached at $4.2 \mathrm{keV}$, just after launch it was at $3.5 \mathrm{keV}$. The upper energy limit of JEM-X has not been affected by the change in gas gain since this limit is determined by the gradually diminishing absorbtion cross section of the Xenon gas above $25 \mathrm{keV}$ and by the sharp K-edge at $35 \mathrm{keV}$. In order to economize on the telemetry usage the detectors are currently operated with an upper threshold set by software to around $50 \mathrm{keV}$.

The detector energy resolution is primarily determined by the number of free electrons liberated during the $\mathrm{X}$-ray absorbtion process and therefore is not significantly affected by the reduction of the gas gain. The energy resolution of the $\mathrm{Xe} \mathrm{K}_{\alpha}$ fluorescence line which is detected all over the sensitive area is still 9.0\% in both JEM-X1 and JEM-X2. 

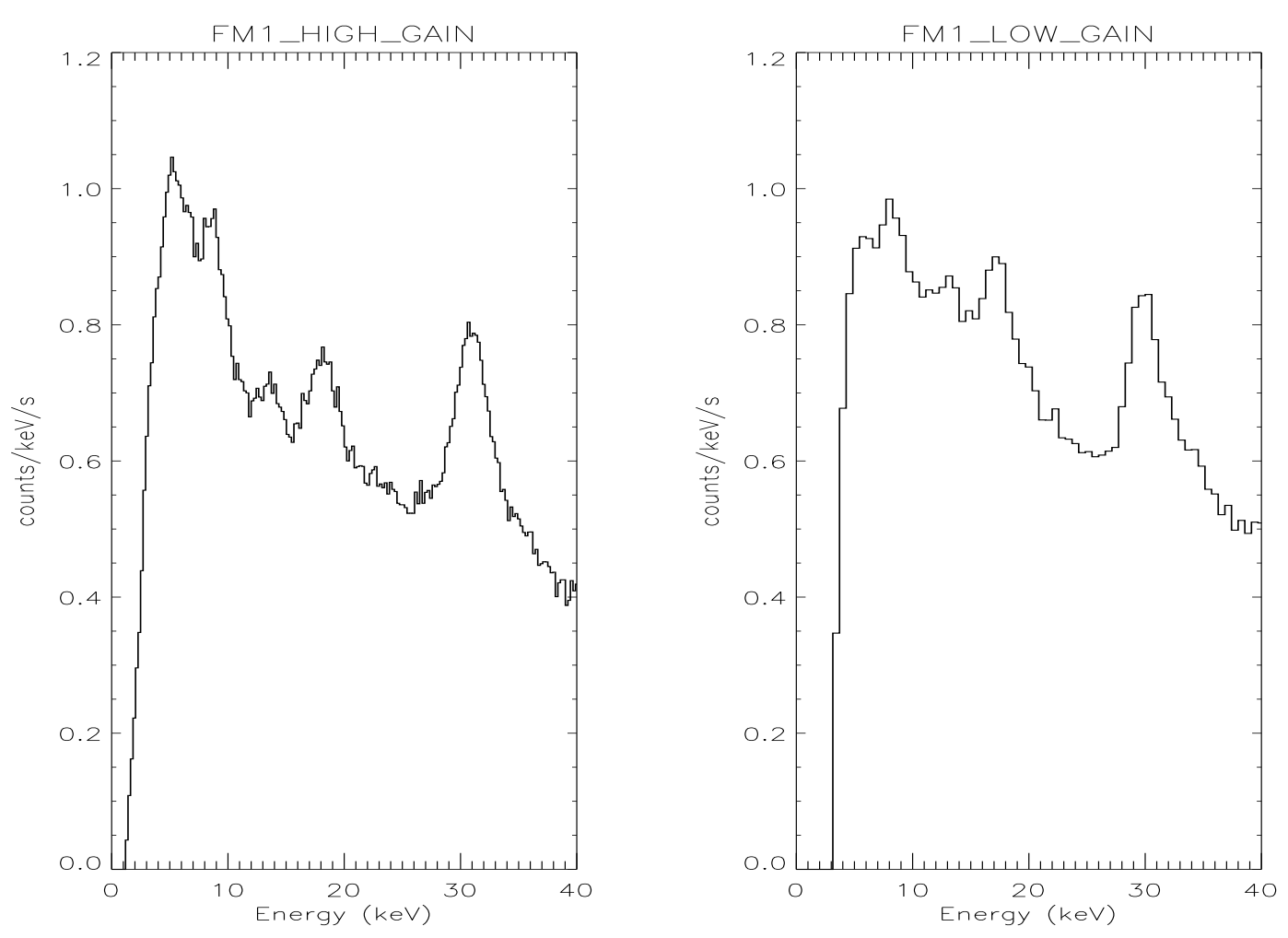

Figure 4. Empty field background spectrum measured with the nominal detector gain of 1500 (left) compared to the background spectrum obtained with the reduced gain of 450. (right)

\subsection{Photon Localization}

The gas gain decrease has led to a degradation of the detector position resolution. The position resolution is, however, still sufficient to resolve the hole pattern of the JEM-X coded mask. Fig. 5 displays the mask shadow gram obtained from an on-axis observation of Cyg X-1 in the energy band from 3 to $35 \mathrm{keV}$. Fig. 6 displays the derived telescope 2D Point Spread Function (PSF). The detector coordinates (DETX, DETY) are given in mm and it should be noted that $1 \mathrm{~mm}$ in the detector plane corresponds to 0.99 arcmin for the JEM-X telescope. The PSF is well represented by a 2D Gaussian function with standard deviations of $\sigma_{x}=1.45 \mathrm{~mm}$ and $\sigma_{y}=1.45$ $\mathrm{mm}$. The JEM-X PSF was analyzed as function of X-ray energy. Fig. 7 displays the energy dependence of the widths and $\sigma_{y}$. The increase of the widths towards lower energies reflects the decrease of the detector position resolutions as the signal-to-electronic noise ratio decreases. However, it must be underlined that the detector resolution is sufficient to achieve useful PSFs across the full 3-35 keV band. An optimal data analysis must take the energy dependence of the PSF into account.

The JEM-X source location accuracy is excellent. INTEGRAL has for calibration purposes made extensive observations of the Crab Nebula region. These observations provided an opportunity to demonstrate the good stability and the statistical precision of the source location with JEM-X. It is known from several X-ray imaging studies of the Crab complex, e.g. Pelling et. al (1987) ${ }^{11}$ that the pulsar is offset in position by 10 to 20 arcsec from the centroid of the nebular emission. JEM-X has made multiple position determinations for the total Crab signal, phase resolved according to the pulsar period. And, as shown in Fig. 8 the derived position actually oscillates in phase with the pulsar signal. The angular displacement between the pulsar and the nebula is 14.4 arcsec for emission in the $3-8 \mathrm{keV}$ band (shown) and decreases to 10 arcsec in the $13-25 \mathrm{keV}$ band. The statistical uncertainty of the individual data points is about 0.5 arcsec. 


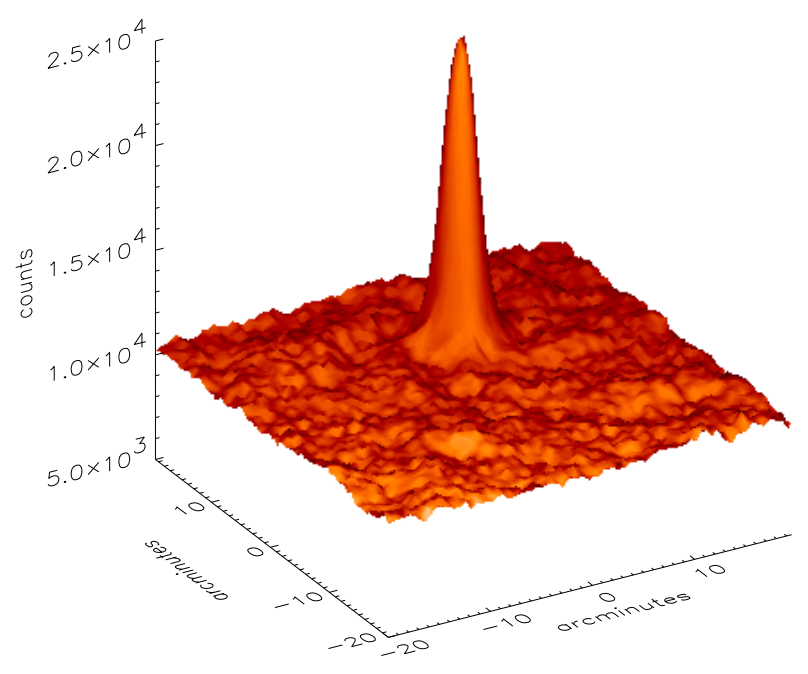

Figure 5. Shadowgram of Cyg $X-1$ (on-axis) in JEM$X 1$ in energy range $3-35 \mathrm{keV}$.

Figure 6. Derived PSF of Cyg $X-1$ (on-axis) in JEM$X 1$ from data shown in Fig. 5.

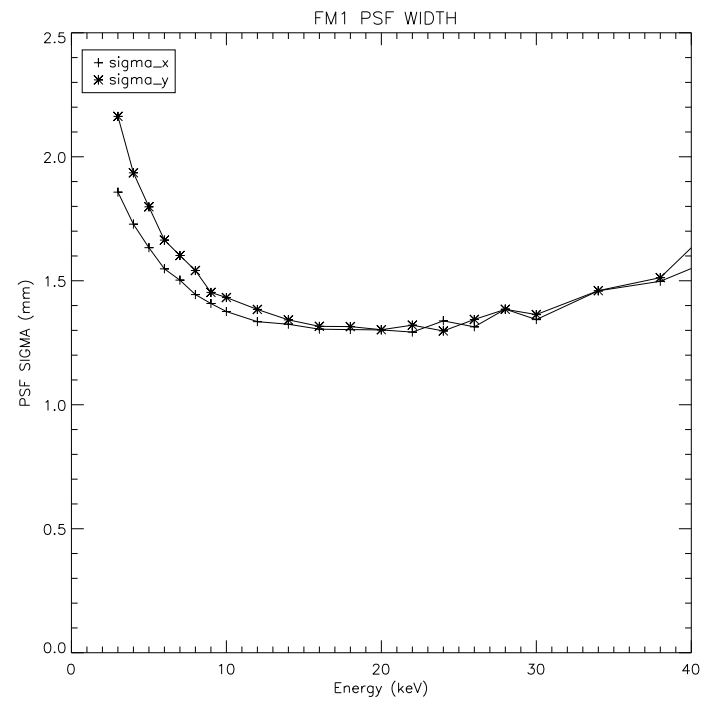

Figure 7. Width of the PSF as a function of energy. 


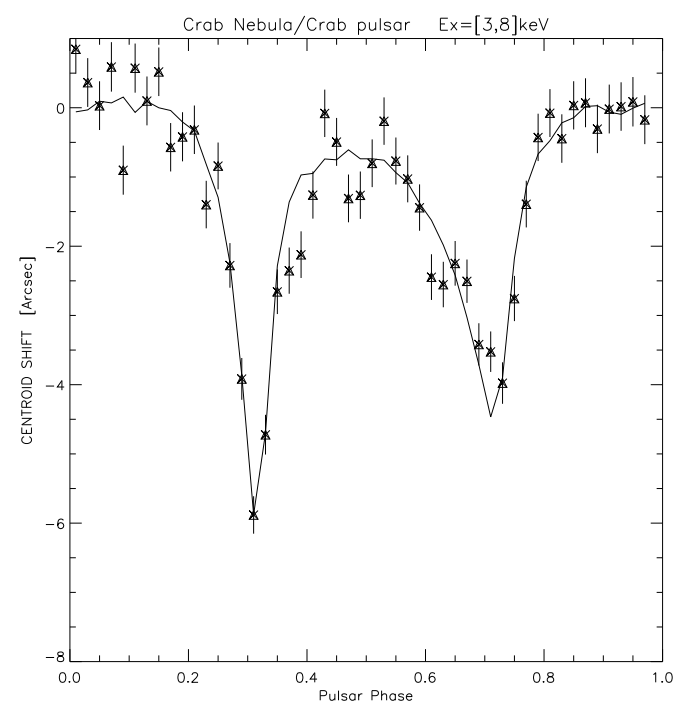

Figure 8. Phase resolved determination of the centroid of the 3 to $8 \mathrm{keV}$ emission from the Crab complex. The separation of the pulsar position from the centroid of the nebular emission results in a "wiggling" of the position in phase with the pulsar signal. The full line is a fit to the data assuming an angular shift between pulsar and nebula of 14.4 arcsecond.

\subsection{Background}

The JEM-X background has been derived from from a number of empty field observations. The background rate is about $20 \mathrm{cts} / \mathrm{s}$ in the 4 to $35 \mathrm{keV}$ range when INTEGRAL is outside the radiation belts. This is about a factor of two higher than predicted before launch.

Fig. 4 illustrates the background energy spectra measured under these conditions. The lines seen here were also observed during the ground calibrations and stem from $\mathrm{Cu}$, Mo and Xe fluorescence and a weak $\mathrm{U}$ contamination of the Be window.

The radial variation in the background across the detector has a noticeable increase towards the edge of the detector.

Table 2 compares the pre-launch predictions, M. Feroci et al.(1999) ${ }^{12}$ for the background count rates with the actual values observed in flight. The count rate data for the Crab (pulsar + nebula) are also shown for reference.

Table 2. JEM-X predicted and actual count rates

\begin{tabular}{|l|l|l|l|l|l|l|l|l|}
\hline $\begin{array}{l}\text { Interval } \\
(\mathrm{keV})\end{array}$ & \multicolumn{2}{|c}{$\begin{array}{c}\text { Source } \\
\text { (counts/s) }\end{array}$} & $\begin{array}{c}\text { DXB } \\
\text { (counts/s) }\end{array}$ & \multicolumn{2}{c}{$\begin{array}{c}\text { CR }^{\dagger} \\
\text { (counts/s) }\end{array}$} \\
\hline & predict & actual & predict & actual & predict & actual & predict & actual \\
\hline $3(4)-10$ & 147 & $(83)$ & 4.1 & $(3.0)$ & 0.8 & $(3.1)$ & 4.9 & $(6.1)$ \\
\hline $10-20$ & 37 & 27 & 2.4 & 1.8 & 1.1 & 5.1 & 3.5 & 6.9 \\
\hline $20-35$ & 7.4 & 5.4 & 0.6 & .5 & 2.3 & 6.5 & 2.9 & 7.0 \\
\hline \hline Tot: 3(4) - 35 & 191 & $(115)$ & 7.1 & $(5.3)$ & 4.2 & $(14.7)$ & 11.3 & $(20.0)$ \\
\hline
\end{tabular}

Presently, it is believed that the background of hard X-and gamma rays produced by cosmic rays in the surrounding payload elements is significantly higher than expected. The increase of the background towards the 
edge of the detector is rather natural if the background arises from photons generated in material around the JEM-X detectors; such an increase would be more difficult to understand if the background was caused by direct traversals of the detector volume by cosmic rays.

When the Sun is active and the satellite altitude is such that the Sun illuminates the JEM-X masks the background in JEM-X may temporarily increase substantially. These situations are, however, relatively rare and only few observations are affected by this problem. It is believed that X-rays from the Sun are scattered into the detector by the mask and its support structure.

So far there has been no indication of a significant long term increase in the background due to radiation activation of the detectors or their surroundings. The monitoring of the background orbital and pointing dependencies will be continued.

\section{JEM-X SENSITIVITY}

The sensitivity of a coded mask instrument like JEM-X is critically dependent on the software used to analyze the data - much more so than for simpler types of X-ray instruments. The sensitivity figures quoted here should not be considered as the final ones - even after six months of intensive work we are still well aware of shortcomings in our analysis software.
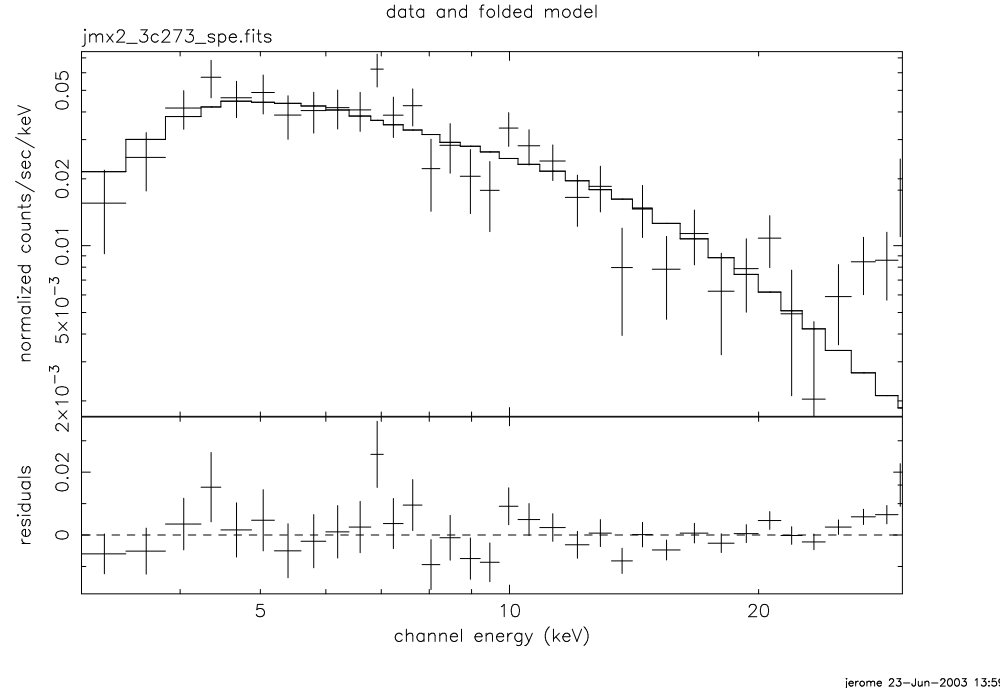

Figure 9. JEM-X observation of 3C273, courtesy T. J.-L. Courvoisier, 2003

During the INTEGRAL Galactic Plane Scan observations (2200 s each) sources down to 20 mCrab are reliably detected when they are inside the central $10^{\circ}$ of the JEM-X field of view. In the same observations many weaker sources - down to $3 \mathrm{mCrab}$ - are detected if they are within the central few degrees of the field of view. These numbers refer to observations with a single JEM-X unit.

Extraction of spectral information from weak sources require more extended observations. Fig. 9 illustrates the capabilities of JEM-X. The displayed spectrum of 3C273 - a 5 mCrab source for JEM-X - was obtained using $22 \mathrm{ksec}$ of on-axis staring. Also these data refer to one JEM-X unit only.

\section{CONCLUSIONS}

The commissioning and performance verification of the two JEM-X instruments on INTEGRAL has been carried out. The operability has been verified and the spectral and spatial resolution has been found to be fully consistent with the values given prior to launch. The source localization capabilities actually significantly exceed the values quoted previously. 
The larger than expected sensitivity of the microstrip detectors to the space environment (presumably induced by the heavy cosmic rays) has threatened to limit the lifetime of the instruments unacceptably. This has forced us to reduce the operating voltage and therefore the gas gain of the microstrip detectors. Even at the reduced gas gain we still experience occasional anode destruction, and therefore it has been decided to operate with only one JEM-X unit until the long term damage rate is better understood.

At the reduced gas gain our energy range has been reduced slightly, the lower threshold ( $50 \%$ efficiency point) has moved up from $3 \mathrm{keV}$ to $4 \mathrm{keV}$.

Taken together, the higher background and operation with only one JEM-X will, at least for the first part of the mission, lead to a reduction of the JEM-X sensitivity by about a factor two compared to the values predicted before launch.

\section{REFERENCES}

1. V.Kämäräinen et al., Proceedings 2nd INTEGRAL Workshop, SP-382, p. 655, 1997.

2. C. Budtz-Jørgensen et al., Proceedings 2nd INTEGRAL Workshop, ESA SP-382, p. 615, 1997.

3. F. Ballesteros et al., Proceedings 2nd INTEGRAL Workshop, SP-382, p. 659, 1997.

4. J. J. M. in't Zand, J. Heise, and R. Jager $A \&$ \& 288, p. 665, 1994.

5. L. D. Baumert, Lecture Notes in Mathematics No 1822: Cyclic Difference Sets, Springer, Berlin, 1971.

6. B. D. Ramsey, R. F. Elsner, and M. C. Weisskopf Nucl. Instr. and Meth. A270, p. 178, 1988.

7. T. Hott Nucl. Instr. and Meth A408, p. 258, 1998.

8. V. Peskov, B. Ramsey, and P. Fonte IEEE Trans. Nucl. Sci. 45, p. 244, 1998.

9. C. Pelliciari, M. de Paoli Vitali, F. Frontera, V. Carassiti, F. Evangelisti, C. Budtz-Jørgensen, and N. J. Westergaard, "The ground calibration apparatus of the JEM-X instrument aboard INTEGRAL," in Proceedings of the Fourth INTEGRAL Workshop, ESA SP-459, pp. 657 - 660, 2001.

10. N. J. Westergaard, C. Budtz-Jørgensen, and N. Lund, "JEM-X Science Performance Report," Tech. Rep. IN-PL-JEM-007, DSRI, www.dsri.dk, June 2002.

11. R. M. Pelling, W. S. Pacies, L. E. Petterson, K. Makishima, M. Oda, , Y. Ogawara, and L. E. Peterson Ap. J. 319, p. 416, 1987.

12. M. Feroci, M. Rapisarda, E. Costa, F. Lei, C. Ferguson, N. J. W. A. J. Dean, and C. Budtz-Jørgensen, "The Background Simulation for the INTEGRAL X-ray monitor JEM-X," in Proceedings of the Third Integral Workshop, 39, p. 429, 1999. Special Issue of Astro. Lett. and Communications. 